\title{
Quality Assurance Measures in the CONCOR National Registry and DNA-Bank of Patients with Congenital Heart Disease in the Netherlands
}

\author{
ET van der Velde ${ }^{1}$, JWJ Vriend ${ }^{2}$, E Theeuwes ${ }^{2}$, HDM Murk Jansen ${ }^{2}$, J Kuiper $^{2}$, WHGJ Hoekstra ${ }^{2}$, \\ BJM Mulder ${ }^{3}$
}

${ }^{1}$ Leiden University Medical Center, Leiden, ${ }^{2}$ Interuniversity Cardiology Institute of The Netherlands, Utrecht, ${ }^{3}$ Amsterdam Medical Center, Amsterdam, The Netherlands

\begin{abstract}
A national registry and DNA-bank of adults with congenital heart disease has been instigated in the Netherlands to investigate long term outcome and the molecular basis of congenital heart defects. All eight academic medical centers in the Netherlands are participating in the CONCOR-project (CONgenital CORvitia).

To assure the quality of the data in the registry, a large number of quality control (QC) measures have been implemented. Some of the items that are checked are: incomplete entries, correct patient-ID and birth-date, possible duplicate patient entries and correctness of registered events.

The CONCOR registration has started in January 2002. Presently (September 2003) 2750 adult patients with congenital heart disease are registered; from $58 \%$ of these patients DNA has already been obtained. The quality checks that have been implemented improve and assure the accuracy of the contents of the registry.
\end{abstract}

\section{Introduction}

Survival of patients with congenital heart disease has dramatically improved since surgical repair became available three decades ago. Instead of a mortality of $85 \%$ during childhood following the natural course, over $85 \%$ of these infants are now expected to reach adulthood [1]. These successes have lead to a new patient population consisting of adults with a surgically corrected heart defect. Although the survival results are gratifying, cure is seldom achieved, and long term complications occur frequently and may require indefinite follow up [2-4]. The number of adults with congenital heart disease in the Netherlands is estimated to be around 20,000 to 25,000 . This number plus an additional 25,000 pediatric patients gives an estimated total of 50,000 patients with congenital heart disease in the Netherlands.

\subsection{CONCOR}

Exact data on long-term outcome of postoperative lesions in this population are not available because a national registry has been lacking. In 2000, the Interuniversity Cardiology Institute of the Netherlands (ICIN) and the Netherlands Heart Foundation instigated a national registry and DNA-bank of patients with congenital heart disease, named CONCOR (CONgenital CORvitia). The aims of this project are: to facilitate investigation of long-term outcome of specific lesions and their treatment, to develop an efficient organizational structure for the improvement of healthcare for patients with congenital heart disease, and to allow investigation of the molecular basis of congenital heart defects. All eight academic medical centers in the Netherlands are participating in the CONCOR project. Participation of the pediatric cardiologists and non-academic hospitals will be pursued as soon as possible.

\subsection{Privacy and security issues}

Data are collected in accordance with the Netherlands and European Privacy Protection Laws. Investigators who request data and DNA for a specific study have to follow a stringent procedure before data and DNA are delivered to the investigator.

\section{Methods}

\subsection{Registration}

After informed consent has been obtained clinical data of adult patients with congenital heart disease are collected using a web-based application (Promise). For each patient, all clinical events (diagnoses and interventions) are registered using the EPCC (European Pediatric Cardiac Code) coding scheme. Blood samples of participating patients are collected, and DNA is isolated and stored. Data is collected by trained research nurses from the patient's clinical records and by interviewing the patients. 


\subsection{Quality Control}

To assure the quality of the data in the CONCOR registry, a large number of quality assurance measures have been implemented.

All data that are collected are stored in a central database using the Promise internet application (http://www.clinicalresearch.nl). One of the strong features of the Promise system is the unlimited quality control during data entry, based on logical criteria. Various queries on the CONCOR database have been designed to create reports based on the content of the registry. For instance, a monthly report is published in the Netherlands Heart Journal based on these data [5]. A printed report of the data entry is kept in the patient's records allowing the cardiologist to easily review the patient's medical history and correct it where necessary.

For quality assurance, a number of additional queries are carried out monthly to identify possible errors or inconsistencies. Some of the items that are checked are:

- Incomplete patient records

- Duplicate (patient) entries

- Correctness of patient-ID and birth-date

- Agreement between the information in the DNAlab database and the CONCOR database

- Overall consistency

Furthermore, from at random selected patient records all data that has been entered (specifically the correct coding of the events) is checked by a trained physician.

\section{Results}

\subsection{General results}

The CONCOR registration has officially started in January 2002. Initially only adults with congenital heart disease are registered. Pediatric patients are expected to be included as off 2004. The number of patients included has increased steadily (see Figure 1).

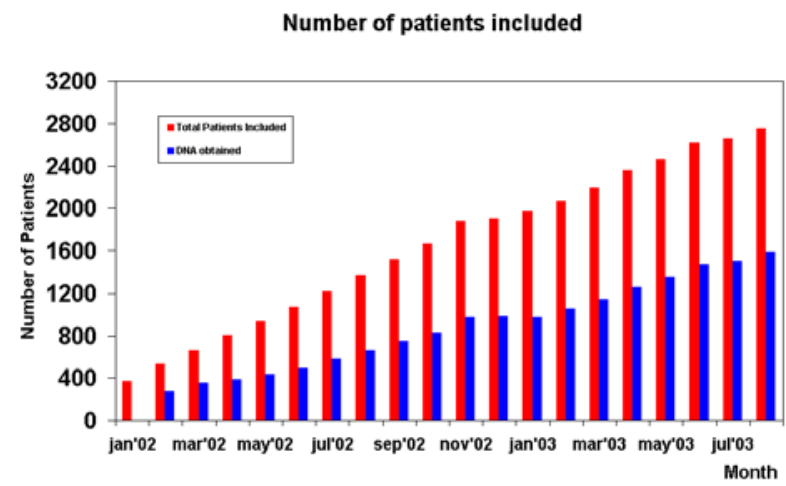

Figure 1. Inclusion results (number of patients, and number of patients where DNA was obtained) per month.
Presently (September 2003) 2750 (adult) patients with congenital heart disease are registered in the CONCOR database. From $58 \%$ of these patients DNA has already been obtained. Table 1 shows the 8 most frequent main diagnoses in these patients. As can be seen, Tetralogy of Fallot is the most frequently occurring underlying disease in these patients, followed by Aortic Coarctation, Aortic Stenosis and Transposition of the Great Arteries.

Presently, $75 \%$ of the patients in the CONCOR registry are between 20 and 40 years of age [Figure 2].

Table 1. Most frequent main diagnoses

\begin{tabular}{ll}
\hline Main diagnosis & $\begin{array}{l}\text { Number } \\
\text { of patients }\end{array}$ \\
\hline Tetralogy of Fallot & 439 \\
Aortic coarctation & 270 \\
Aortic valvar stenosis & 254 \\
$\begin{array}{l}\text { Complete transposition of the } \\
\text { great arteries }\end{array}$ & 247 \\
$\begin{array}{l}\text { Pulmonary valvar stenosis } \\
\text { (congenital) }\end{array}$ & 173 \\
ASD II & \\
VSD & 131 \\
Marfan syndrome & 127 \\
\hline
\end{tabular}

Figure 2. Age distribution (\%) and percentage of patients (per age group) with arrhythmias.

\section{CONCOR Age distribution \& arrhythmia}

Age distribution (\%) $\square$ \% with Arrhythmia

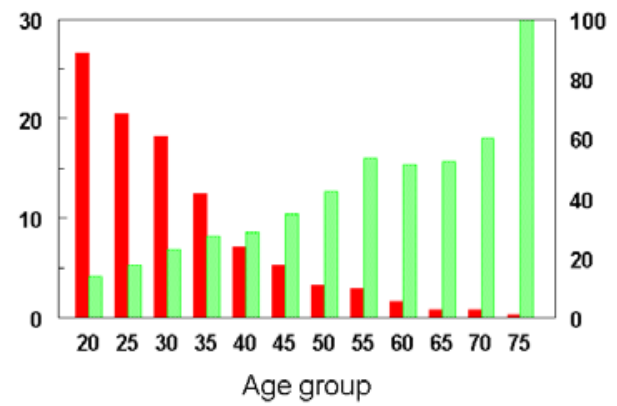

\subsection{Quality Control}

In Figure 2, some of the results of the monthly quality assurance (QA) queries are shown: the number of patients with incomplete records, and the number of duplicate entries. Both numbers have decreased to zero or almost zero since the start (in January 2003) of the Quality Control (QC) efforts. 


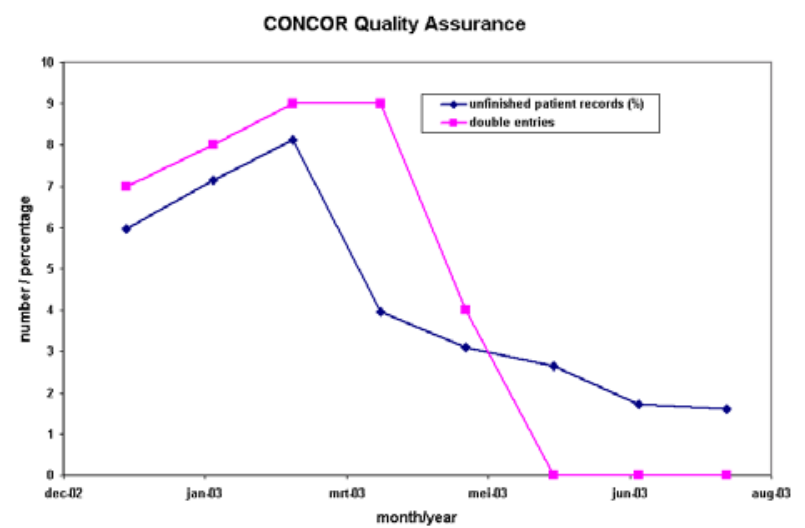

Figure 3. Percentage of incomplete patient records and number of duplicate records in the CONCOR registry per month.

\subsection{DNA laboratory quality control}

Blood samples of participating patients are collected, and DNA is isolated and stored at the DNA-lab facilities of the Amsterdam Medical Center (AMC). The DNA-lab only receives (with the blood samples) only a CONCOR record-number and patient's birth date to prevent that DNA can be traced back to a specific patient without the CONCOR registry database.

At regular instants (bi-monthly), the DNA-lab database (containing only CONCOR record number and birth date) are compared with the CONCOR registry database in order to identify possible mismatches. The DNA-lab also warns the CONCOR project-coordinator if for a specific patient not enough DNA could be obtained from the blood samples; in that case, the research nurses are notified that from that patient blood should be collected again.

\subsection{Quality control actions and effects}

If the QC results show that action is necessary, the QC manager issues a QC modification document which describes the problem and the actions to be taken (usually by the research nurses).

Results from the QC queries have also in a number of cases lead to:

- changes to the clinical data

- slight changes in the data entry work process

- slight changes in the database structure

\section{Discussion}

The population of patients with congenital heart disease is steadily growing especially due to the succesful developments in the field of cardiovascular surgery. Long-term results on outcome, however, are not available. At present, $75 \%$ of the patients in the CONCOR registry are between 20 and 40 years of age
[Figure 2]. Moreover, it also has become clear that children of patients with congenital heart disease have a higher risk of congenital heart defects than children of normal individuals [6]. In the last few years the genetic aspects of congenital heart disease have increasingly become a field of interest for research [7]. So far little is known about the genetic, molecular basis of congenital heart disease but more and more genes are identified to play a role in the development of the heart. These facts necessitate augmentation of genetic research in patients with congenital heart disease.

Registration and collection of DNA of patients with congenital heart disease are important tools to facilitate research on long term outcome and to allow investigation of the molecular basis of congenital heart defects. The availability of a national registry is mandatory for scientific research and management [8]. Regular checks of accuracy and consistency of the data in the registry are essential to assure a high quality of data and DNAcollection.

\section{Conclusion}

The quality checks that have been implemented have been shown to improve and assure the accuracy of the contents of the CONCOR registry. Furthermore, based on these results, improvements in the CONCOR database have been implemented.

\section{Acknowledgements}

This project is supported by grants from the Netherlands Heart Foundation (2001T501) and the Interuniversity Cardiology Institute in the Netherlands (ICIN).

\section{References}

[1] Perloff JK. Congenital heart disease in adults. A new cardiovascular subspecialty. Circulation 1991;84:1881-90.

[2] Kaplan S, Perloff JK. Survival patterns after surgery or interventional catherisation. In: Perloff JK, Child JS (eds). Congenital Heart Disease in Adults. Philadelphia: WB Saunders Company, 1998: p 54.

[3] Stark J. Do we really correct congenital heart defects? J Thorac Cardiovasc Surg 1989; 97:1.

[4] Vriend JWJ, Van der Velde ET, Mulder BJM. Adults with congenital heart disease: a growing population. Netherlands Heart Journal 2002;10:345-347.

[5] CONCOR newsletter (monthly). Netherlands Heart Journal 2003;11:322.

[6] Burn J, Brennan P, Little J, et al. Recurrence risks in offspring of adults with major heart defects: results from first cohort of British collaborative study. Lancet 1998;351:311-16.

[7] Perloff JK, Warnes CA. Challenges posed by adults with repaired congenital heart disease. Circulation 2001;103:2637-2643.

[8] British Cardiac Society Working Party. Grown-up 
congenital heart $(\mathrm{GUCH})$ disease: current needs and provision of service for adolescents and adults with congenital heart disease in the UK. Heart 2002;88:11i14.
Address for correspondence.

Enno T. van der Velde, MSEE, PhD

Medical Physicist

Department of Cardiology

Leiden University Medical Center

P.O. BOX 9600, 2300 RC Leiden

The Netherlands

E-mail address: ETvanderVelde@lumc.nl 